\title{
Determining Dominant Frequency with Data-Adaptive Windows
}

\author{
Gagan Mirchandani and Shruti Sharma \\ School of Engineering, College of Engineering \& Mathematical Sciences, \\ University of Vermont
}

\begin{abstract}
Measurement of activation rates in cardiac electrograms is commonly done though estimating the frequency of the sinusoid with the greatest power. This frequency, commonly referred to as Dominant Frequency, is generally estimated using the short-time Fourier Transform with a window of fixed size. In this work a new short-time Fourier transform method with a data-adaptive window is introduced. Experiments are conducted with both synthetic and real data. Results for the former case are compared with current state-of-the-art methods. Given the difficulty in identifying activation points in electrograms, experiments reported in the literature have so far used only synthetic data. The new method is tested by application to real data, with true activation rates determined manually. Substantial improvement is observed. An error analysis is provided.
\end{abstract}

Keywords: atrial fibrillation, data-adaptive windows, non-stationary signals, dominant frequency.

\section{Introduction}

Measurement of temporal beat-to-beat variations in heart rate is one of the key factors in the determination of cardiac disorders. In atrial fibrillation (AF), the atria contract rapidly and irregularly. The corresponding heart rate, measured through intracardiac electrograms, shows rapidly changing frequencies between 3-15 Hz. Since AF can be associated with heart disease and heart failure, an accurate determination of heart rate is essential for proper management purposes. Activation rate or frequency, is defined as the inverse of the time period between two consecutive activation points. While manual determination of atrial activation timings by experts is theoretically possible, the sheer volume of data that would need analysis make this an impractical option. Accordingly, automated methods for determining the beat-to-beat variations have received much attention. This is a difficult task for many reasons: electrogram data is random and nonstationarity and there is no clearly identifiable spectral estimation technique that specifically matches the particular problem of atrial activation rate determination. Methods using the short-time Fourier transform (STFT) suffer from the usual time duration, frequency resolution problem. Furthermore, complexity in identifying activation time, often due to morphology fragmentation, further complicates the spectral estimation task.

A. Elmoataz et al. (Eds.): ICISP 2010, LNCS 6134, pp. 287-296, 2010.

(C) Springer-Verlag Berlin Heidelberg 2010 
There currently do not exist automated methods for the determination of activation rates. Using synthetic data and fixed-window STFT analysis, results have been reported for the so-called dominant frequency (DF) associated with some specific structures of the data. DF is defined as the frequency with the greatest power in the power spectral estimate of data. In this paper we introduce a new STFT-based method for power spectral estimation of electrogram data. A data-adaptive window is employed to partially mitigate the resolution problem. The method is tested using both synthetic data as also real electrogram data where the true activation rates are determined manually. Results with the fixed-window and the new data-adaptive window are compared. An error analysis shows substantial gains with the data-adaptive window method. The latter method can also find use in other similar non-stationary data applications such as eeg power spectral estimation.

\section{State of the Art in Electrogram Analysis}

Methods used for determining AF include time-frequency analysis [1], 11, hidden Markov models [15], deconvolution [3, and spectral analysis, with the latter method being the predominant one. Motivated perhaps by the hypothesis that sites of high frequency activation might serve as drivers for fibrillation and could therefore be targets for ablation therapy [13, [16, spectral estimation has centered around frequency domain methods and specifically around the concept of DF within the framework of a fixed window STFT [6], 12, [13, 14, [16], [5], 10, , 9], 7]. While for certain synthetic signals, DF correlates well with the mean, mode and median of the activation time [12, 6], results with real data show poor correlation [4]. Surprisingly, there are very few results in the literature reporting on the accuracy of the DF methodology applied to signals, real or synthetic. For those cases that are documented, synthetic signals are created by placing a Hanning window to emulate a clean morphology [13, 6]. Another interesting approach for the understanding of AF is in the use of morphology matching for the determination of the direction of progression of the activation wavefront. Cross-correlation is used to determine the recurrence of a morphology and that information is used to detect a pattern in the flow of the electrical activity [2] 8 .

\section{Spectral Analysis Using Variable Windows}

As indicated earlier, DF characterizes electrogram data with varying frequency, by the one frequency with the largest power in the power spectral density. However, given that the signal is non-stationary, it is clear that for an accurate determination of activation rates, electrogram signals should best be analyzed locally to capture the changing nature of the frequencies. As a solution, a dataadaptive window method is proposed: the window length is determined by the time resolution required for the signal. Hence ideally, with the frequency between $3-15 \mathrm{~Hz}$, the spectrogram data should be analyzed in segments with a maximum 


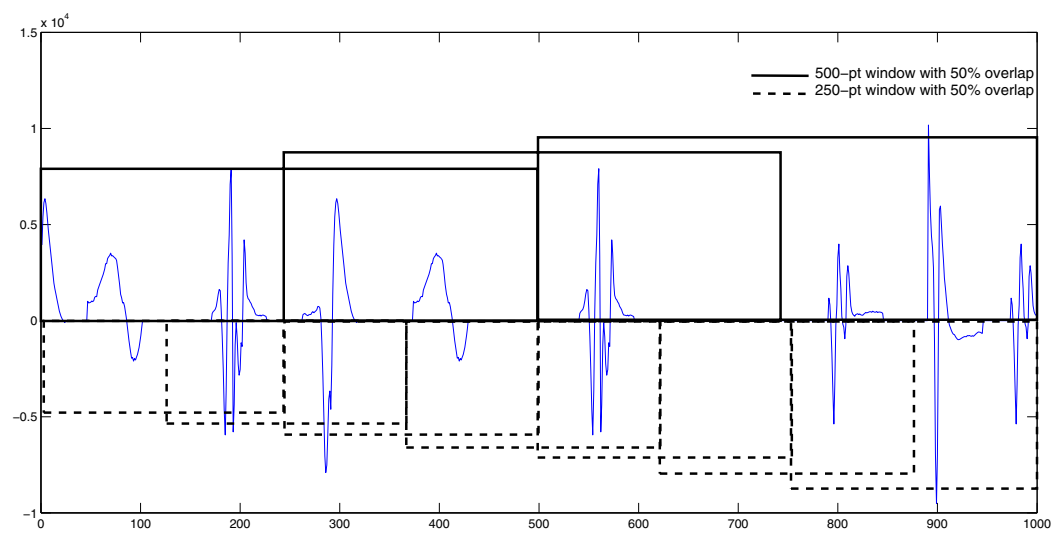

Fig. 1. Synthetic signal

of two activations per segment. Accordingly, given that a $f \mathrm{~Hz}$ signal exhibits $f$ activations (peaks) per second, the ideal size window for a $4 \mathrm{~Hz}$ signal would be 500 points, decreasing linearly to 125 points for a $16 \mathrm{~Hz}$ signal, assuming 1 $\mathrm{kHz}$ sampling rate. However, the small windows are designed to analyze each segment with a maximum of three activations in each segment ( 3 activations in a $250 \mathrm{~ms}$ window would mean an inter-event frequency of $12 \mathrm{~Hz}$ ) and since the inter-event period would not be greater than $250 \mathrm{~ms}$ (the total length of the segment being analyzed is $250 \mathrm{~ms}$ ), the signal was not analyzed in segments of $125 \mathrm{~ms}$.

In the proposed method the DF is first determined using the STFT with a 500point window with a $50 \%$ overlap. If the observed DF is greater than $4 \mathrm{~Hz}$, a 250 point window (also with a $50 \%$ overlap) is utilized. Dominant frequencies obtained from each of the overlapping windows are then used to label frequencies over 125 ms segments of the signal, to effectively generate a localized frequency estimate. To illustrate the technique, the method is applied to a simple electrogram signal where the signal allows for 8 125-ms DF estimates over the 1000-ms duration.

Example 1: The synthetic signal shown in Figure 1, with randomly changing frequency between $3-15 \mathrm{~Hz}$ was created using specific morphologies that were obtained by pacing the heart from different directions. The signal was analyzed using the data-adaptive windows described above.

For the $1000 \mathrm{~ms}$ signal shown in Figure 1, a 500-point window with the $50 \%$ overlap provides 3 consecutive signal segments. The DF for each of the 3 segments shows frequencies greater than $4 \mathrm{~Hz}$. Accordingly, 250-point windows are utilized. With the $50 \%$ overlap, there are 6 consecutive 125 -ms signal segments, for which the DF is determined. These, as also the true activation rates (determined manually) are placed on a frequency-time plot and compared. Note that the latter frequencies occur at actual activation times and are shown accordingly 
Table 1. Ground truth activation rates and DF estimate using data-adaptive windows

\begin{tabular}{|c|c|c|}
\hline \multicolumn{3}{|c|}{ Activation rates (ground truth) } \\
\hline \begin{tabular}{l|l|l|} 
& 8 & 10
\end{tabular} & \begin{tabular}{|l|l|l|l|}
9 & 6 & 4 & 11 \\
\end{tabular} & 11 \\
\hline \multicolumn{3}{|c|}{ Data adaptive windows } \\
\hline $500 \mathrm{~ms}$ & \multicolumn{2}{|c|}{ Windows - 250ms Overlap } \\
\hline \begin{tabular}{|l|l|}
11 \\
\end{tabular} & \begin{tabular}{|l|l|}
8 & 10 \\
\end{tabular} & \\
\hline \multicolumn{3}{|c|}{$250 \mathrm{~ms}$ Windows - 125ms Overlap } \\
\hline \multicolumn{3}{|c|}{\begin{tabular}{|l|l|l|l|l|l|l|l|}
10 & 11 & 10 & 7 & 5 & 4 & 10 & \\
\end{tabular}} \\
\hline \multicolumn{3}{|c|}{ DF estimate using both windows } \\
\hline \begin{tabular}{|l|l|l|}
10 & 10 & 11 \\
\end{tabular} & \begin{tabular}{|l|l|l|l|}
10 & 7 & 5 & 4 \\
\end{tabular} & 10 \\
\hline
\end{tabular}

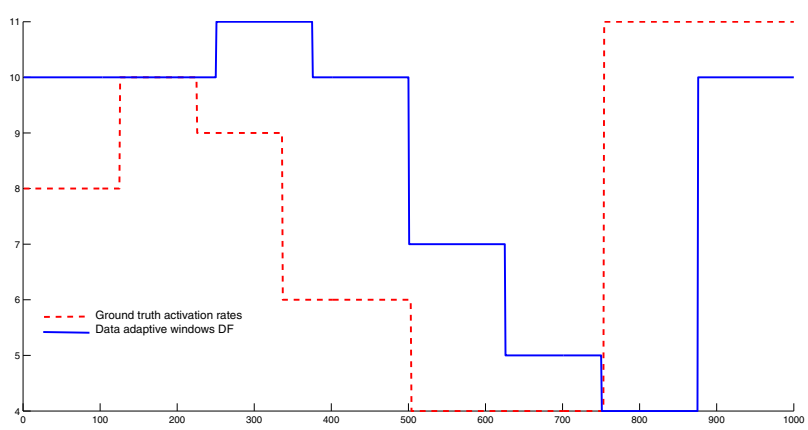

Fig. 2. DF error using data-adaptive windows

on the frequency-time plot. The DF estimates however, using the two fixed-size adaptive windows, occur at activation times that are integer multiples of 250 or $125 \mathrm{~ms}$. The final frequency estimates are obtained by using the $250 \mathrm{~ms}$ window results and extrapolating the start and end-points to cover the whole range from 0 to $1000 \mathrm{~ms}$. Hence for the example here, the frequency over the 1-250 ms and for $875-1000 \mathrm{~ms}$ range are extrapolated to $5.3 \mathrm{~Hz}$, and $3 \mathrm{~Hz}$ respectively.

The activation rates and their DF estimates, while generated over different sized ms-blocks, are assumed to apply over each ms, generating a time series, shown in Figure 2 that can be used for error analysis.

\section{Experiments and Results}

Experiments were divided into two categories: (i) those similar to ones in the reported literature (ii) those with real morphologies.

\subsection{Previous Experiments}

The first set of experiments were conducted to compare results reported in [12], referred to here as the fixed-window method. In that work, electrogram data was analyzed in segments of 4000 points. Segments were created using a 50-point 
Hanning window as the morphology, while the frequencies were chosen using a mean frequency from a uniform distribution between $3-9 \mathrm{~Hz}$, with a standard deviation of $1 \mathrm{~Hz}$. Four sets of tests were conducted with the following conditions:

1. Constant amplitude, constant frequency.

2. Varying amplitude, constant frequency.

3. Constant amplitude, varying frequency.

4. Varying amplitude, varying frequency.

Since the first two tests involved constant frequency, these experiments were not duplicated since a variable window analysis would not provide any gain. The last two sets of tests were conducted with synthetic signals. For the purpose of this paper, one hundred sets of 4000 point signals were created where the frequency was varied randomly between 3-10 Hz. A 50-point Hanning window was used to depict a filtered, rectified biphasic morphology. The average interevent frequencies, and the DF estimates from the data-adaptive window method were then compared to the DF estimates using the fixed-window method.

Figures 3(a) and 4(a) show the sample synthetic signals with varying frequency where the peak amplitude ratio was constant (condition 3) and where it was varied between 0.1 and 1 (condition 4). These are followed by the scatter plots of the DF by the fixed-window method vs. average inter-event frequency (ground truth) and the DF by the data-adaptive window method vs. average inter-event frequency (ground truth). The correlation coefficient for the tests using the fixed-window and the data-adaptive window methods were determined to be 0.069 and 0.91 for test 3 , and 0.071 and 0.81 for test 4 respectively.

\subsection{Experiments Using Real Morphologies}

In order to simulate signals that resemble real electrograms, signals were generated by pacing the heart manually from different directions. Seven different morphologies were generated. Each morphology was placed on a baseline with an inter-event frequency chosen at random between $3-10 \mathrm{~Hz}$ to create 10,000 point synthetic signals. These were analyzed using data-adaptive windows. The mean and standard deviation of the error was determined.

The signals was subject to the standard electrogram preprocessing procedures:

1. Band-pass filtering between $40-250 \mathrm{~Hz}$.

2. Signal rectification.

3. Low-pass filtering between $0-20 \mathrm{~Hz}$

4. Window the signal with a Hamming window.

Experiments were conducted using both rectification and squaring, the latter as an alternative to rectification. DF obtained using the data-adaptive window method for both were formulated as time-series with each frequency value representing as before, frequencies over $1 \mathrm{~ms}$ for the $125 \mathrm{~ms}$ segments. These were then compared to the ground-truth values. The error series for five synthetic signals 
a.

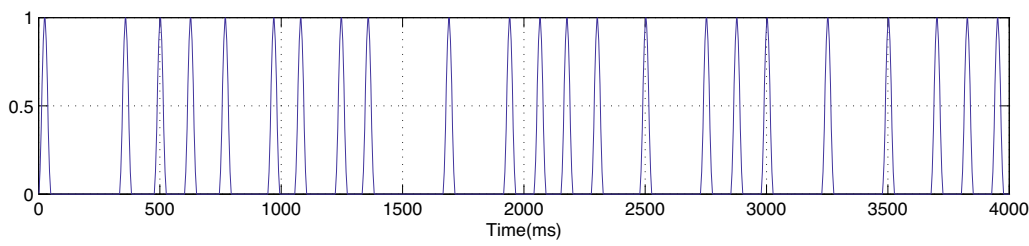

b.

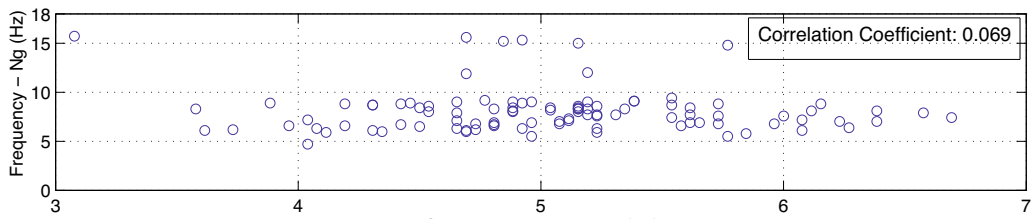

Ground Truth Frequency $(\mathrm{Hz})$

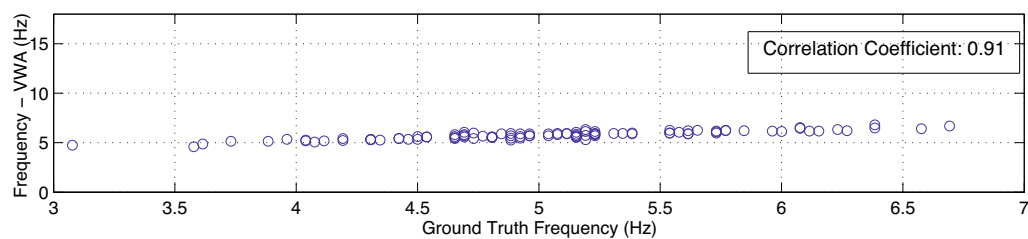

Fig. 3. (a). Sample signal with constant amplitude and variable frequency. (b) DF using fixed-window vs average inter-event frequencies (c) DF using data adaptive windows vs average inter-event frequencies.

a.

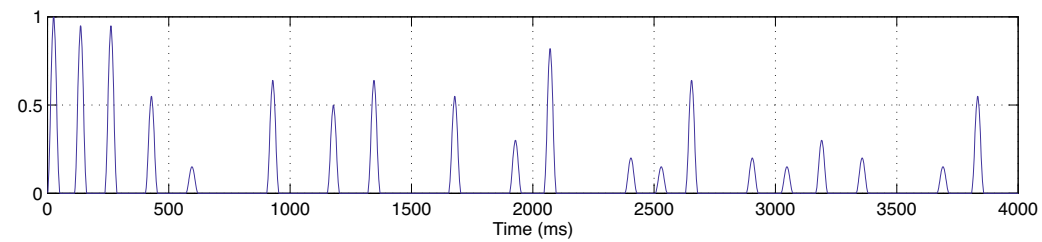

b.
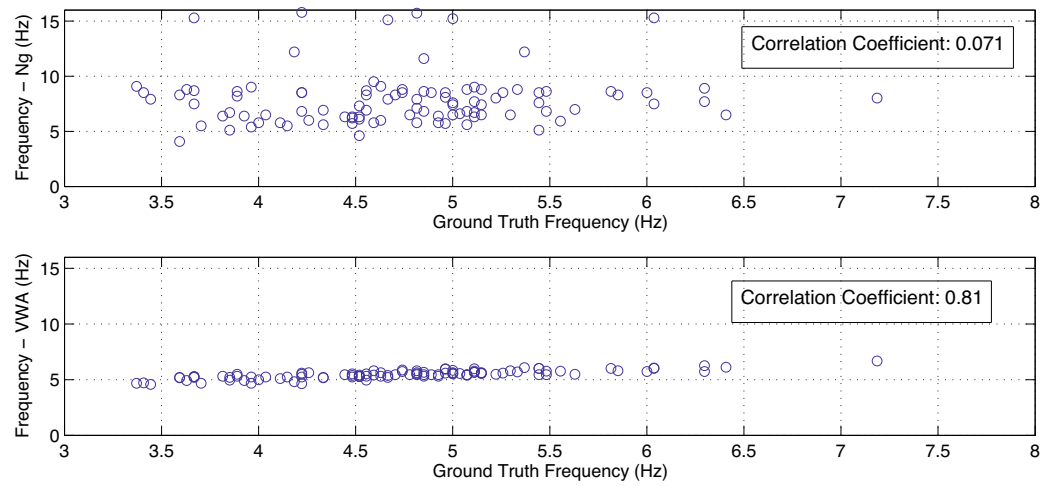

Fig. 4. (a). Sample signal with variable amplitude and variable frequency (b) DF using fixed-window vs average inter-event frequencies (c) DF using data adaptive windows vs average inter-event frequencies. 

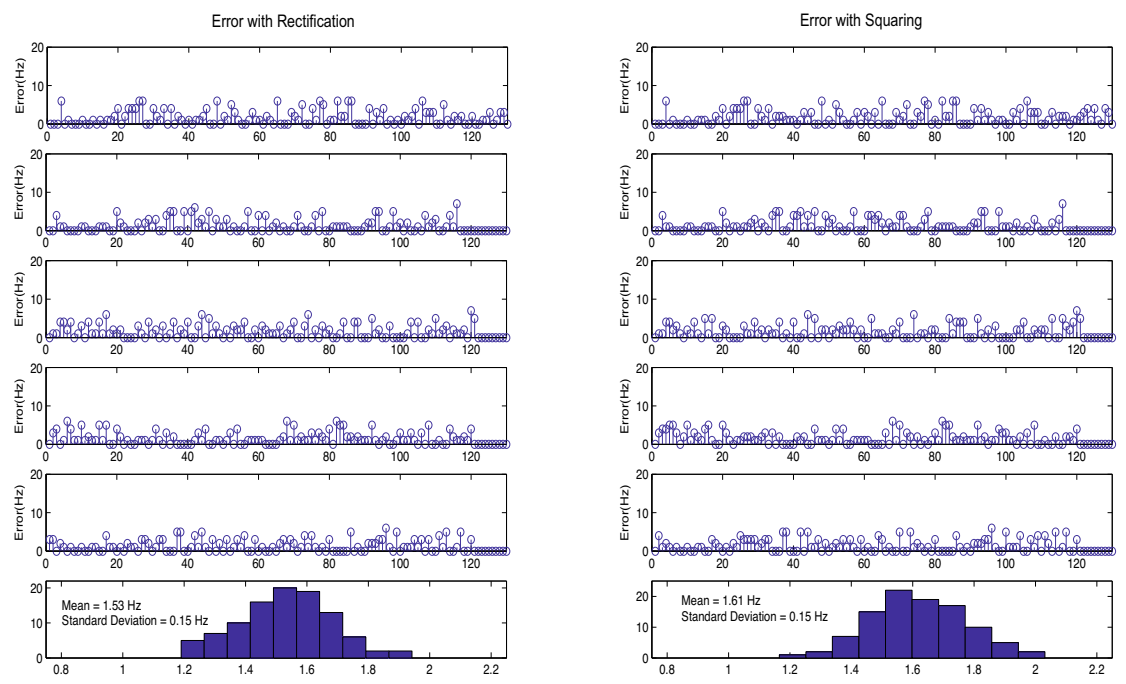

Fig. 5. Error and error distribution with the data-adaptive method using real morphologies
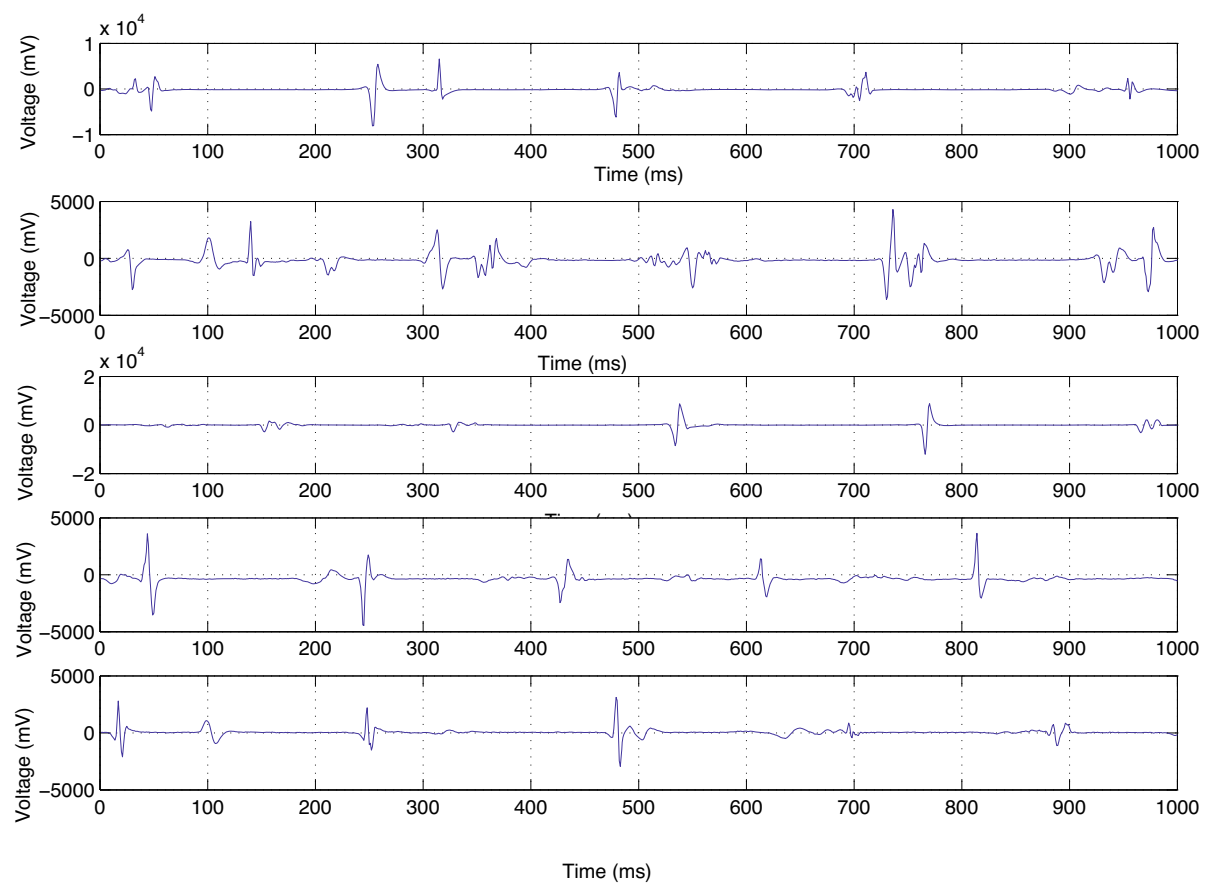

Fig. 6. Examples of real signals from real AF electrograms that were used for the experiments 

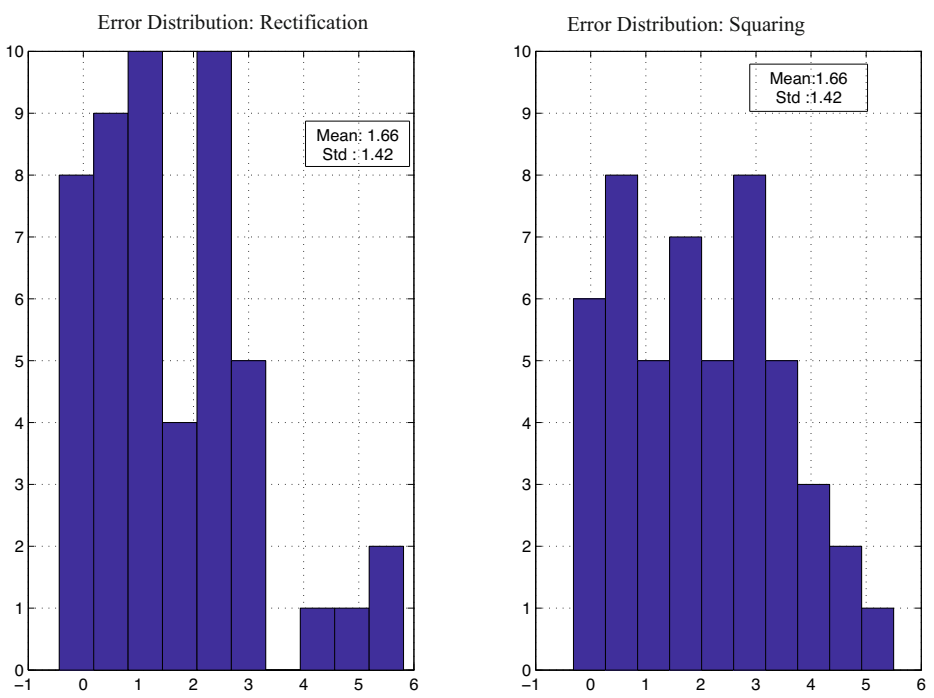

Fig. 7. Error distribution with rectification and squaring, using real data

are shown in Figure 5. Also shown is the error distribution for the average error of each of the hundred synthetic signals created. The mean errors for squaring and rectification were $1.50 \mathrm{~Hz}$ and $1.61 \mathrm{~Hz}$ with a standard deviation of $0.15 \mathrm{~Hz}$ for both respectively.

\subsection{Experiments Using Real Signals}

Fifty sets of 1000-point real signals were obtained from a patient with AF and are shown in Figure 6. Activation times and rates were determined manually. Signals were preprocessed and analyzed using the data-adaptive window method. It is seen that data-adaptive window method was able to capture the changing frequency of the signals with a mean error of $1.66 \mathrm{~Hz}$ and standard deviation of 1.42 $\mathrm{Hz}$ for both squaring and rectification. Error distribution is shown in Figure 7.

\section{Conclusion}

A data-adaptive window has been used with the STFT to capture non-stationary characteristics of synthetic signals representing electrogram data. Tests were conducted using 100 sets of 4000-point data with variable frequency and fixed amplitude and variable frequency and variable amplitude. Correlating results with the ground truth shows the data-adaptive and fixed-window methods performing with correlation coefficients 0.91 and 0.069 for the first case and 0.81 and 0.071 respectively for the second. Experiments with real morphologies with the data-adaptive window showed a mean error of 1.50 and $1.61 \mathrm{~Hz}$ and standard deviation of $0.15 \mathrm{~Hz}$. Using 50 sets of real 1000-point electrogram data showed a mean error of $1.66 \mathrm{~Hz}$ and standard deviation of $1.42 \mathrm{~Hz}$. 


\section{Acknowledgements}

Support from a Graduate Teaching Fellowship from The University of Vermont and a Research Fellowship from Medtronics is hereby acknowledged.

\section{References}

1. Addison, P.S., Watson, J.N., Clegg, G.R., Steen, P., Robertson, C.E.: Finding coordinated atrial activity during ventricular fibrillation using wavelet decomposition. IEEE Engineering in Medicine and Biology 58-65 (January/February 2002)

2. Barbaro, V., Bartolini, P., Calcagnini, G., Censi, F., Michelucci, A., Morelli, S.: Mapping the Organization of human atrial fibrillation using a basket catheter. Computers in Cardiology, 475-478 (1999)

3. Ellis, W.S., Eisenberg, S.J., Auslander, D.M., DAe, M.W., Zakhor, A., Lesh, M.D.: Deconvolution: A novel signal processing approach for determining activation time from fractionated electrograms and detecting infarcted tissue. Circulation 94, 2633-2640 (1996)

4. Elvan, A., et al.: Dominant Frequency of Atrial Fibrillation Correlates Poorly with Atrial Fibrillation Cycle Length. Circulation, Arrhythmia and Electrophysiology 2, 634-644 (2009)

5. Everett, T.H., Kok, L.-C., Vaughn, R.H., Moorman, J.R., Haines, D.E.: Frequency domain algorithm for quantifying atrial fibrillation organization to increase defibrillation efficacy. IEEE Transactions on Biomedical Engineering 48(9), 69-978 (2001)

6. Fischer, G., Stühlinger, M.C., Wieser, B., Nowak, C.-N., Wieser, L., Tilg, B., Hintringer, F.: On Computing Dominant Frequency From Bipolar Intracardiac Electrograms. IEEE Transactions on Biomedical Engineering 54(1), 165-169 (2007)

7. Le Goazigo, C.: Measurement of the dominant frequency in atrial fibrillation electrograms. MSc. Thesis, Cranfield University (2005)

8. Houben, R.P.M., Allessie, M.A.: Processing of intracardiac electrograms in atrial fibrillation. IEEE Engineering in Medicine and Biology Magazine, 40-51 (November/December 2006)

9. Jacquemet, V., Oosterom, A.V., Vesin, J.V., Kappenberger, L.: Analysis of electrocardiograms during atrial fibrillation. IEEE Engineering in Medicine and Biology Magazine, 79-88 (November-December 2006)

10. Langley, P., Bourke, J.P., Murray, A.: Frequency analysis of atrial fibrillation. Computers in Cardiology, 65-68 (September 2000)

11. Moghe, S.A., Qu, F., Leonelli, F.M., Patwardhan, A.R.: Time-frequency representation of epicardial electrograms during atrial fibrillation. Biomedical Sciences Instrumentation 36, 45-50 (2000)

12. Ng, J., Kadish, A.H., Goldberger, J.J.: Effect of electrogram characteristics on the relationship of dominant frequency to atrial activation rate in atrial fibrillation. Heart Rhythm 3(11), 1295-1305 (2006)

13. Ng, J., Goldberger, J.J.: Understanding and interpreting dominant frequency analysis of AF electrograms. Journal of Cardiovascular Electrophysiology 18(7), 680-685 (2007) 
14. Ng, J., Kadish, A.H., Goldberger, J.J.: Technical considerations for dominant frequency analysis. Journal of Cardiovascular Electrophysiology 18(7), 757-764 (2007)

15. Sandberg, F., Stridth, M., Sörnmo, L.: Frequency tracking of atrial fibrillation using hidden Markov models. IEEE Transactions on Biomedical Engineering 55(2), 502$511(2008)$

16. Sanders, P., Berenfeld, O., Hocini, M., Jaïs, P., Vaidyanathan, R., Hsu, L.-F., Garrigue, S., Takahashi, Y., Rotter, M., Sacher, F., Scavëe, C., Ploutz-Snyder, R., Jalife, J., Haïssaguerre, M.: Spectral analysis identifies sites of high frequency activity maintaining atrial fibrillation in humans. Circulation 112, 789-797 (2005) 